\section{Further Comments}

The invariant temperatures 1280 and $1330{ }^{\circ} \mathrm{C}$ were taken from the work of [72Ere2], as was the estimated eutectic composition, 58 at.\% $\mathrm{Pd}$. In the process of drawing the diagram, however, it became clear that the (metastable) congruent melting point of $\mathrm{Ti}_{2} \mathrm{Pd}_{3}$ would lie above the liquidus if 58 at.\% Pd were used for the eutectic composition. At that point, a more explicit error estimate should have been attached to the eutectic composition, which should have been tabulated as $56 \pm 2$ at. $\%$ Pd. Based on published thermal analysis, metallographic, and X-ray data, it does not appear possible to be more precise than this. For example, a lever rule calculation of the eutectic composition requires the composition of $\alpha \mathrm{TiPd}$, which is not precisely determined.

In this phase diagram, as in Ti-Ir, Ti-Rh, and Ti-Pt, there is much less information than meets the eye. To plot the diagram, we require quite definite numbers and the uncertainties in those numbers are not represented in the finished graph.

Above reply by

Joanne L. Murray

National Bureau of Standards

$* * *$

We invite your comments on this or any other topic. - Editor

\section{Addenda}

\section{The Pd-Ti (Palladium-Titanium) System}

Corrections for Table 1 on page 322 of Vol. 3, No. 3 and further comments on the $\mathrm{Pd}-\mathrm{Ti}$ system are given in the Comments section (see above).

\section{The Nd-Sc (Neodymium-Scandium) System}

In Fig. 1 on page 356 of Vol. 3, No. 3, the high-temperature BCC solid solution phase should also be identified as " $(\beta \mathrm{Nd}, \beta \mathrm{Sc})$ ". The same addition should be made to the atomic percent tear-out on page 391 and the weight percent tear-out on page 392 .

\section{Recent Literature}

Thanks are due to Dr. William G. Moffatt for calling attention to the following correction: on page 570 of Vol. 3 , No. 4, the entry under TI-V (and the title of the article) should be Ti-V.

\section{The Al-Be (Aluminum-Beryllium) System}

Figures 1 and 2 on pages 50 and 52 of Vol. 4, No. 1 were inadvertently reversed; the full diagram should appear as Fig. 1, and the diagram extending from 0 to 0.4 at. $\% \mathrm{Be}$ should appear as Fig. 2. The tear-outs on pages 99-102 are correctly identified.

\section{Calendar of Events}

\author{
October 2-6, \\ 1983
}

\section{TMS-AIME Fall Meeting}

Location: Philadelphia, PA, U. S. A. Sponsor: TMS-AIME

Among the sessions planned are Metal-Gas Systems, chaired by N. A. Gokcen, and Evaluation and Representation of Alloy Phase Diagrams, chaired by L. H. Bennett. Both sessions, which are sponsored by the ASM-MSD Alloy Phase Diagram Data Committee, will be held on Monday, Oct 3 in Salon 3 at Franklin Plaza. Subjects to be presented in the morning session are "Metal-Oxygen Systems" by C. Altstetter, "Phase Equilibria in Selected Binary Transition Element-Nitrogen Systems" by N. A. Gokcen, "Binary Hydrogen Systems" by J.F. Smith, "Thermodynamic Properties of the Ternary Iron-Nickel Monosulfide with NiAs Structure, (Fe, Ni)S" by K.-C. Hsieh, and "Phase Equilibria in Gas-Containing MetalMetal Systems" by R. D. Shull. The afternoon session will include "Strategies on Coupling Phase Diagram and Thermodynamic Data" by C. W. Bale, "Thermodynamic Modeling and Phase Diagram Calculations" by Y. A. Chang, "Computer Representation of Phase Diagrams" by P. Nash, "The Computer Representation of Alloy Phase Diagrams" by D. J. Orser, and "Phase Fraction Charts" by J. E. Morral. For further information, contact Marilyn Zabel, The Metallurgical Society of AIME, 420 Commonwealth Drive, Warrendale, PA 15086, U.S. A.; telephone: 412/776-9050.

\section{November 14-17, 1983}

Phase diagrams may be discussed in several of the symposia, such as those on thin films and interfaces, ion implantation and ion beam processing of materials, and rapidly solidified metastable materials. For more information, contact the MRS Secretariat, 110 Materials Research Laboratory, University Park, PA 16802, U.S.A.

\section{February 26- March 1, 1984}

\section{Annual Meeting of the American Institute of Mining. Metallurgical, and Petroleum Engineers (AIME)}

Location: Los Angeles, CA, U.S. A. Sponsor: AIME

This symposium on Metastability in Alloys, jointly sponsored by the MSD-ASM Thermodynamics and Alloy Phase Diagram Data Committees at the 1984 AIME Annual Meeting in Los Angeles, will cover theoretical and experimental aspects of thermodynamics of metastable phases, coherent phase diagrams, and other aspects of constrained equilibria in alloy systems. For additional information, contact Marilyn Zabel, The Metallurgical Society of AIME, 420 Commonwealth Drive, Warrendale, PA 15086, U.S. A.; telephone: 412/776-9050. 